\title{
The clinical efficacy of dabigatran etexilate for preventing stroke in atrial fibrillation patients
}

\author{
This article was published in the following Dove Press journal: \\ Vascular Health and Risk Management \\ 8 July 2013 \\ Number of times this article has been viewed
}

\section{Christopher R Ellis \\ Daniel W Kaiser}

Vanderbilt Heart and Vascular Institute, Nashville, TN, USA
Correspondence: Christopher R Ellis Vanderbilt Heart and Vascular Institute, I2I I 2 Ist Ave, South Suite 5209, Nashville, TN, USA 37232-8802

Tel + I 6I59362523

Email christopher.ellis@vanderbilt.edu
Abstract: The use of novel oral anticoagulants (NOACs) for stroke and systemic embolism prevention in the setting of specifically non valvular atrial fibrillation has provided clinicians with a realistic treatment alternative to the traditional dose-adjusted, warfarin-based anticoagulation that is targeted to a therapeutic international normalized ratio range of 2.0-3.0. We discuss the use of dabigatran in the setting of mechanical heart valves, atrial fibrillation or left atrial catheter ablation procedures, reversal of the drug in the setting of adverse bleeding events, and background on the molecular biology and development of this novel treatment for stroke reduction.

Keywords: NOACs, systemic embolism, atrial fibrillation, stroke, dabigatran etexilate

\section{Introduction}

The use of novel oral anticoagulants (NOACs) for stroke and systemic embolism prevention in the setting of specifically non valvular atrial fibrillation (NVAF) has provided clinicians with a realistic treatment alternative to the traditional doseadjusted, warfarin-based anticoagulation that is targeted to a therapeutic international normalized ratio (INR) range of 2.0-3.0. The focus of this review will be to evaluate published data on the clinical efficacy of dabigatran etexilate in preventing stroke and systemic embolism in patients with NVAF. We will discuss the use of dabigatran in the setting of mechanical heart valves, atrial fibrillation (AF) or left atrial catheter ablation procedures, reversal of the drug in the setting of adverse bleeding events, and background on the molecular biology and development of this novel treatment for stroke reduction.

\section{Mechanism of action}

Intravenous direct thrombin inhibitors (DTI) were first approved in 1998, with lepirudin being approved for patients with clinically relevant heparin-induced thrombocytopenia. The development of an oral DTI was technically difficult, as DTI are highly polar and lipophilic in nature. ${ }^{1}$ The oral form needs to be a fat-soluble prodrug that is transferred back into the active drug after absorption. Ximelagatran was the first oral DTI with proven efficacy compared to warfarin, but this compound was withdrawn by the company in 2006 due to an increased risk of liver toxicity., ${ }^{2,3}$ Dabigatran etexilate is a synthetic, small-molecule prodrug of the DTI dabigatran. Dabigatran is a univalent DTI that blocks the active catalytic site of thrombin in a competitive and reversible manner. The bivalent DTIs, such as bivalirudin, additionally block exosite 1, the docking site for fibrin, in addition to the active site. ${ }^{4}$ 
Dabigatran blocks the terminal coagulation cascade by binding to the active site of thrombin (factor IIa) in a dose and concentration-dependent fashion (Figure 1). Thrombin plays a central role in blood coagulation by converting fibrinogen into a network of fibrin, amplifying its own generation by feedback activation of factors V, VIII, and XI, and by stimulating platelets. ${ }^{5,6}$ Thrombin also activates factor XIII, which favors the formation of cross-linked bonds among the fibrin molecules, stabilizing the clot. In contrast to the indirect thrombin inhibitors such as heparin and low molecular weight heparin, dabigatran is not dependent on antithrombin III activity. As a result, dabigatran is able to inhibit not only free thrombin, but also clot-bound thrombin, and in theory could be a more effective anticoagulant for active thrombus. ${ }^{7,8}$ Dabigatran directly inhibits coagulase, unlike heparin and hirudin, and thus prevents fibrin formation and may increase the sensitivity of S. aureus to antibiotics. ${ }^{9}$ Furthermore, by reducing the activation of platelets, dabigatran has an antiplatelet effect. ${ }^{10}$

\section{Metabolism}

In order to optimize absorption in the gastrointestinal tract, dabigatran capsules contain a tartaric acid core that produces a local acidic environment. The tartaric acid is thought to cause the dyspepsia side effects, which occur in approximately $10 \%$ of patients. ${ }^{11}$ Dabigatran can be taken with food to decrease dyspepsia, but caution is advised given the limited postmarket experience with dabigatran. ${ }^{12}$ After absorption, dabigatran etexilate is rapidly absorbed and hydrolyzed by serum esterases into its active moiety, dabigatran. The bioavailability is approximately $7 \%$, requiring relatively high dosages for adequate serum levels. Serum concentrations remain relatively static under fast or fed conditions. ${ }^{13}$ Within 2 hours of oral administration, the drug has reached maximal anticoagulant effect, limiting the need for bridging anticoagulation. In postoperative patients, serum levels flattened with delayed peak concentrations (median 6 hours), suggesting a slower rate of absorption in the early postoperative period. ${ }^{14}$ Approximately $20 \%$ is conjugated with glucuronic acid to form a pharmacologically active conjugate; $80 \%$ is excreted by the kidneys unchanged. ${ }^{15}$ In patients with normal renal function, the mean terminal half-life of dabigatran is approximately 8 hours after a single dose and up to 14 hours after multiple doses enabling twice daily (BID) dosing. ${ }^{13,16}$ In patients taking $150 \mathrm{mg} \mathrm{BID}$, the peak and trough plasma concentrations are $180 \mathrm{mg} / \mathrm{mL}$ and $90 \mathrm{ng} / \mathrm{mL}$, respectively. ${ }^{16}$ With creatine clearance $(\mathrm{CrCl})$ of $30 \mathrm{~mL} /$ minute, the terminal half-life doubles to over 24 hours. ${ }^{17}$

\section{Drug interactions: contraindications and interactions}

Major contraindications to dabigatran use include renal impairment ( $\mathrm{CrCl}<15 \mathrm{~mL} /$ minute), advanced liver disease (impaired baseline clotting function), a prosthetic heart valve, or hemodynamically significant heart valve disease. ${ }^{18}$

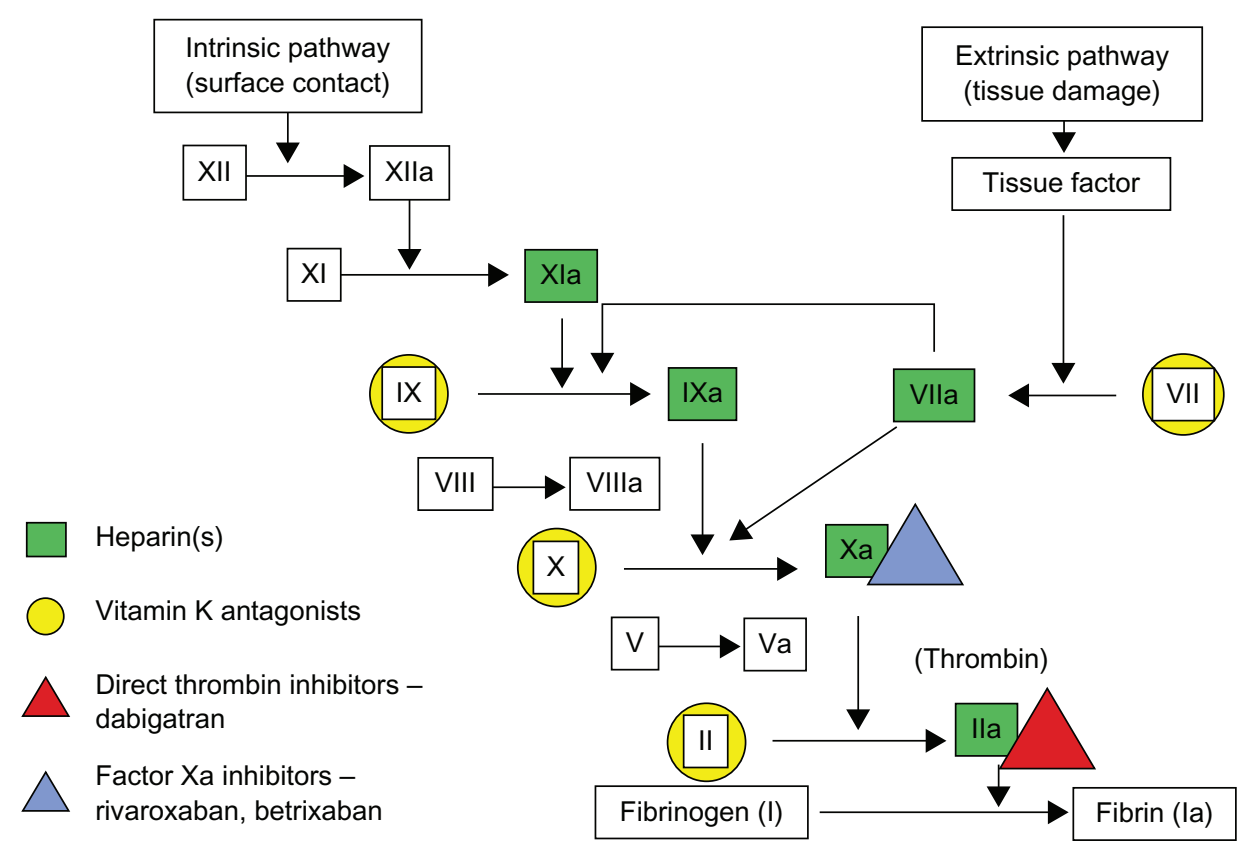

Figure I Detailed visual description of the coagulation cascade and the sites blocked either directly or indirectly by old and newer systemic anticoagulants. Notes: Reprinted with permission Nagarakanti R, Ellis CR. Dabigatran in clinical practice. Clin Ther. 20I2;34(I0):205I-2060.57 
Careful consideration should be made in the setting of medications affecting the permeability $(\mathrm{P})$-glycoprotein pump, gastrointestinal (GI) acidity, and concomitant antiplatelet medications. Fortunately, dabigatran is not metabolized by cytochrome P450 (CYP) and does not affect CYP activity. ${ }^{19}$

\section{Renal and liver dysfunction}

In patients with normal renal function, the standard dosing of dabigatran etexilate is $150 \mathrm{mg}$ BID for patients with NVAF. In patients with a $\mathrm{CrCl}$ of $15-30 \mathrm{~mL} /$ minute, a dose reduction to $75 \mathrm{mg}$ BID dosing is available; note the $75 \mathrm{mg}$ BID dosing is based on in vitro pharmacokinetics of the drug, and has not been formally studied in human clinical trials. Dabigatran should not be used in patients with a $\mathrm{CrCl}<15 \mathrm{~mL} /$ minute or in those who are hemodialysis-dependent. Drug labeling recommends that renal function should be assessed before starting dabigatran at least annually in patients $>75$ years of age or in those with a $\mathrm{CrCl}<50 \mathrm{~mL} /$ minute. In patients with mild to moderate hepatic impairment, there was a slight reduction in bioconversion of the prodrug; however, the total anticoagulant effect was comparable and no dose adjustment is needed for moderate hepatic impairment. ${ }^{20}$

\section{P-glycoprotein pump}

The prodrug dabigatran etexilate (but not the active drug dabigatran) is a substrate of the efflux permeability glycoprotein transporter ( $\mathrm{p}$-Glycoprotein pump) which is highly expressed in the intestines; thus, any potential effects are restricted to drug absorption, particularly if taken shortly before dabigatran etexilate oral administration. ${ }^{16} \mathrm{P}$-glycoprotein functions to pump dabigatran molecules back into the GI tract, such that the inhibitors quinidine, amiodarone, and verapamil, increase plasma concentrations of dabigatran. Coadministration with some inhibitors, such as systemic ketoconazole and dronedarone, approximately doubles dabigatran levels; it is recommended that a dose reduction be made in dabigatran to $75 \mathrm{mg}$ BID for patients with moderate renal impairment ( $\mathrm{CrCl} 30-50 \mathrm{~mL} / \mathrm{minute})$ who are also prescribed these medications. ${ }^{21}$ The only drug that is specifically noted in the dabigatran US package insert (USPI) as contraindicated is the P-glycoprotein inducer rifampin, which reduces plasma concentrations by $66 \% .^{21}$

\section{Proton pump inhibitors and antiplatelet agents}

Oral absorption of dabigatran etexilate is increased in a locally acidic environment. Coadministration of dabigatran with proton pump inhibitors decreases the plasma levels of dabigatran by approximately $25 \% .^{13}$ The clinical implications of this (in terms of stroke reduction and bleeding risk) were not demonstrated in the RE-LY (Randomized Evaluation of Long-Term Anticoagulation Therapy) trial. ${ }^{25}$ Coadministration of dabigatran etexilate with antiplatelet agents should be approached with caution because of an increased risk of bleeding. In the RE-LY study, coadministration of aspirin or clopidogrel increased risk of major bleeding by about twofold. See Figure 2 for a complete summary of food and drug interaction.

\section{Monitoring}

The pharmacokinetic profile of dabigatran etexilate is predictable with low inter and intra individual variability, which permits renal-based dosing without the need for routine monitoring. ${ }^{15,22}$ Numerous tests are available to determine if a patient is taking dabigatran, but available tests to help guide dosing are lacking. Although dabigatran increases the prothrombin time (PT) and INR in a concentration-dependent fashion, the response is unpredictable and should not be used as a measure of anticoagulation. The activated partial thromboplastin time (aPTT), which targets the intrinsic pathway of coagulation, is increased more predictably by dabigatran; however, the aPTT concentration-response curve is curvilinear and flattens at higher concentrations. ${ }^{23}$ While PTT is commonly used to monitor the anticoagulant effect of heparin with or without concomitant warfarin, doing so with therapeutic dabigatran could lead to inaccurate results. There is limited data for the use of activated clotting time (ACT) on dabigatran's anticoagulant effect. The Ecarin clotting time (ECT) is the most sensitive parameter of anticoagulant activity, but it is not generally available in hospital laboratories and has been used largely as a research tool. The most accessible qualitative method for determining the presence or absence of dabigatran's anticoagulant effect is the thrombin time (TT) assay. The commercially available Hemoclot ${ }^{\circledR}$ Thrombin Inhibitor assay (Hyphen BioMed, Neuville-sur-Oise, France) is a diluted thrombin time (dTT) assay sensitive enough for accurate quantitative measurement of dabigatran activity across a broad concentration range (Figure 3 ). ${ }^{24}$

\section{Clinical trial data with dabigatran in patients with NVAF}

Dabigatran etexilate was approved for anticoagulation in patients with NVAF based on the results of the RE-LY trial. ${ }^{25}$ The RE-LY trial was a prospective, Phase III trial which consisted of two blinded doses of dabigatran $(110 \mathrm{mg}$ or 


\begin{tabular}{|c|c|c|}
\hline & Interaction & Clinical considerations \\
\hline Food & $\begin{array}{l}\mathrm{T}_{\max } \text { delayed } \\
\mathrm{C}_{\max } \text { and } \mathrm{AUC} \text { unchanged } \\
\text { Reduced inter-individual variability }\end{array}$ & $\begin{array}{l}\text { No dose adjustment is recommended: can be given with or without } \\
\text { food }\end{array}$ \\
\hline $\begin{array}{l}\text { Absorption } \\
\downarrow \text { dabigatran concentration }\end{array}$ & Antacids, pantoprazole & $\begin{array}{l}\text { Diminished clinical effect may occur, as may be expected for any } \\
\text { drug resulting in an increase in gastric } \mathrm{pH} \text { during dabigatran } \\
\text { administration. Dabigatran should be administered at least } 2 \mathrm{~h} \\
\text { before antacid administration } \\
\text { No dose adjustment is recommended }\end{array}$ \\
\hline \multirow[t]{2}{*}{$\begin{array}{l}\text { P-gp inhibitors } \\
\uparrow \text { dabigatran concentration }\end{array}$} & Amiodarone, veraparmil, quinidine & $\begin{array}{l}\text { No dose adjustment is generally recommended for AF patients, } \\
\text { however, caution should still be used. To minimize potential for } \\
\text { interaction, dabigatran should be given at least } 2 \mathrm{~h} \text { before these } \\
\text { drugs }\end{array}$ \\
\hline & Digoxin & $\begin{array}{l}\text { No dose adjustment is recommended. The combination was safe and } \\
\text { well tolerated }\end{array}$ \\
\hline \multirow[t]{2}{*}{$\begin{array}{l}\text { CYP3A4 and P-gp inhibitors } \\
\uparrow \text { dabigatran concentration }\end{array}$} & Ketoconazole & $\begin{array}{l}\text { Caution is advised when co-administering dabigatran with } \\
\text { ketoconazole }\end{array}$ \\
\hline & Clarithromycin & $\begin{array}{l}\text { No dose adjustment is recommended, however, caution should be } \\
\text { exercised }\end{array}$ \\
\hline $\begin{array}{l}\text { CYP3A4 and P-gp inducers } \\
\downarrow \text { dabigatran concentration }\end{array}$ & Rifampin & $\begin{array}{l}\text { Avoid concurrent use of dabigatran with rifampin if possible. Closely } \\
\text { monitor patients for decreased effects of dabigatran if concomitantly } \\
\text { administering rifampin }\end{array}$ \\
\hline $\begin{array}{l}\text { Antiplatelet agents } \\
\uparrow \text { anticoagulant effect }\end{array}$ & Aspirin, clopidrogrel & $\begin{array}{l}\text { No dose adjustment is recommended. Caution should be exercised } \\
\text { due to added anticoagulant effect }\end{array}$ \\
\hline $\begin{array}{l}\text { LMWH } \\
\uparrow \text { anticoagulant effect }\end{array}$ & Enoxaparin & No dose adjustment is generally recommended. Use with caution \\
\hline $\begin{array}{l}\text { NSAIDs } \\
\uparrow \text { antiplatelet effect }\end{array}$ & Diclofenac & $\begin{array}{l}\text { No dose adjustment is recommended } \\
\text { Monitor for excessive bleeding due to added antiplatelet effect }\end{array}$ \\
\hline $\begin{array}{l}\text { Herbs } \\
\downarrow \text { dabigatran concentration }\end{array}$ & St John's wort & Co-administration of these products is not recommended \\
\hline
\end{tabular}

Figure 2 Summary of food and drug interactions with dabigatran.

Note: Reprinted with kind permission from the Springer Science and Business Media. ${ }^{21}$

Abbreviations: $\mathrm{T}_{\max }$, time to maximum concentration; $\mathrm{C}_{\max }$, maximum plasma concentration; $\mathrm{AUC}$, area under the curve; $\mathrm{P}$-gp, $\mathrm{P}$-glycoprotein; $\mathrm{AF}$, atrial fibrillation; CYP3A4, cytochrome P450 3A4; LMWH, low molecular weight heparin; NSAIDs, nonsteroidal anti-inflammatory drugs.

$150 \mathrm{mg}$ BID) compared with open-label warfarin (target INR 2-3). The primary efficacy outcome in the RE-LY trial was stroke or systemic embolism and the primary safety outcome was major hemorrhage. Secondary outcomes were stroke, systemic embolism, and death. Patients were followed up 14 days after randomization, at 1 and 3 months, every 3 months thereafter for the first year, and then every 4 months until the end of the study.

The study involved 18,113 patients with NVAF and at least one major risk factor for thromboembolism. Risk factors included previous stroke or transient ischemic attack or systemic embolism, left ventricular ejection fraction $<40 \%$ or symptomatic heart failure (New York Heart Association class II or higher in the last 6 months), hypertension, age $>75$ years, or age $65-74$ years with either diabetes mellitus or coronary artery disease. Exclusion criteria included patients who had a prosthetic heart valve or hemodynamically significant valve disease, severe renal failure ( $\mathrm{CrCl}$ less than $30 \mathrm{~mL} /$ minute), active liver disease, disabling or recent stroke, recent or pending surgery, recent or known bleeding disorders, uncontrolled hypertension, need for anticoagulation of disorders other than AF, planned ablation or surgery for AF, reversible causes of AF, or pregnancy. Hemodynamically significant valve disease was defined as heart valve disease of sufficient severity that the patient would be expected to potentially undergo surgical valve replacement during the RE-LY trial study period. The minimum follow-up was 1 year and the median follow-up was 2 years.

Dose adjusted warfarin therapy reached the primary outcome of all stroke (ischemic or hemorrhagic) or systemic embolism rate of $1.71 \%$ per year, with an accompanying major bleeding rate of $3.57 \%$. Dabigatran etexilate, $150 \mathrm{mg}$ BID, reduced the rate of stroke by $34 \%(P<0.001$ for superiority; relative risk (RR): 0.65 ; $95 \%$ confidence interval (CI): $0.52-0.81$ ), and at this dose there was no increase in major bleeding (3.32\%). Dabigatran etexilate, $110 \mathrm{mg}$ BID, had a decreased but similar rate of stroke and systemic embolism (1.54\% per year) that was 
A

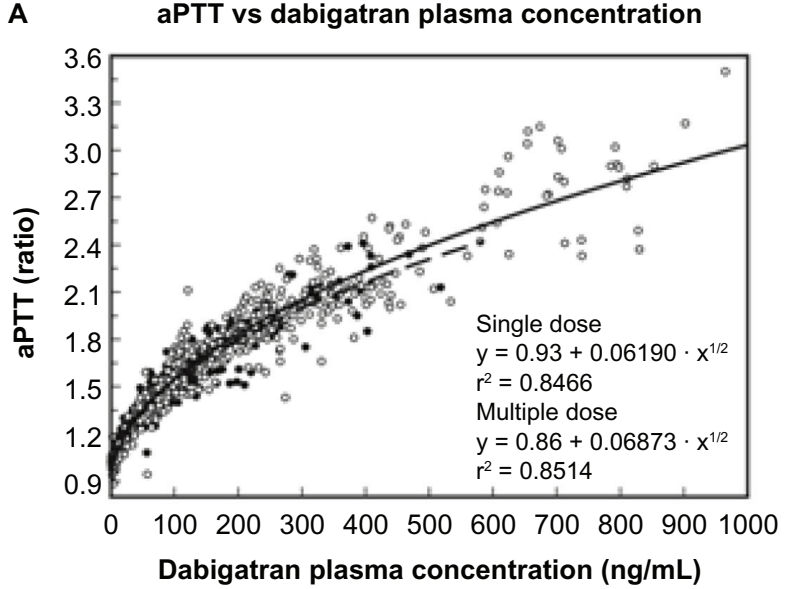

B

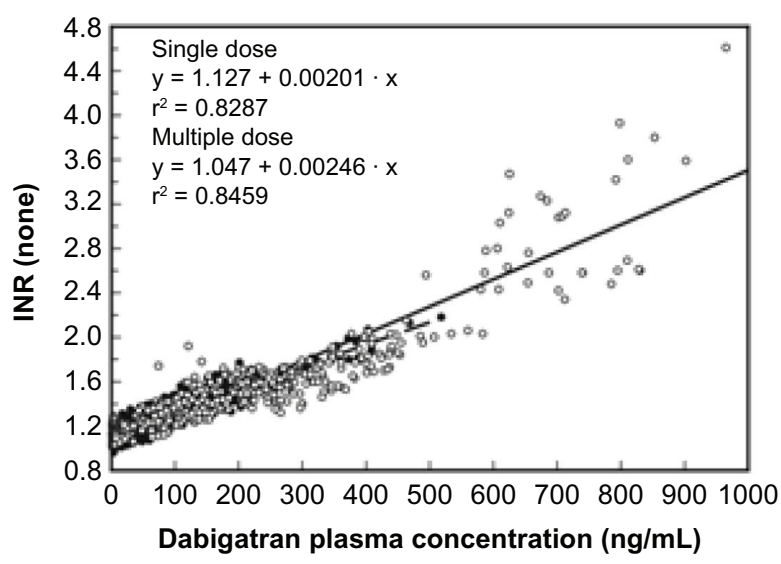

C Thrombin time vs dabigatran plasma concentration

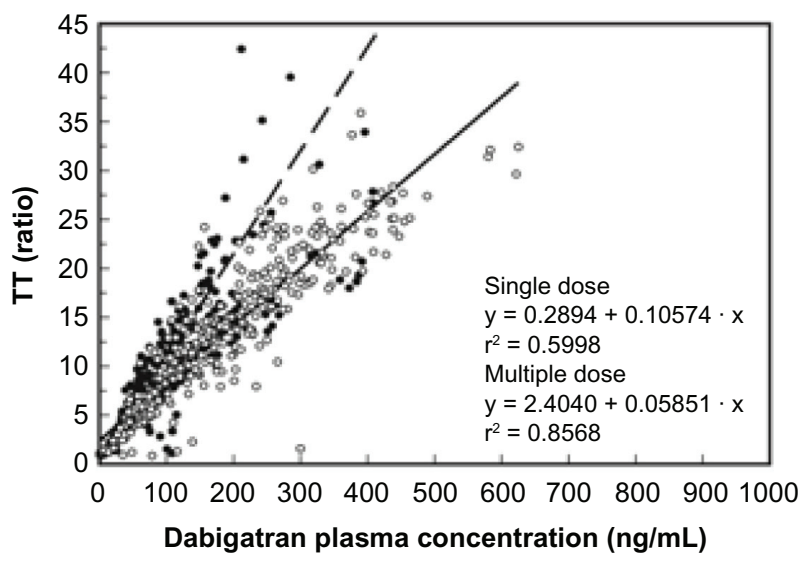

D

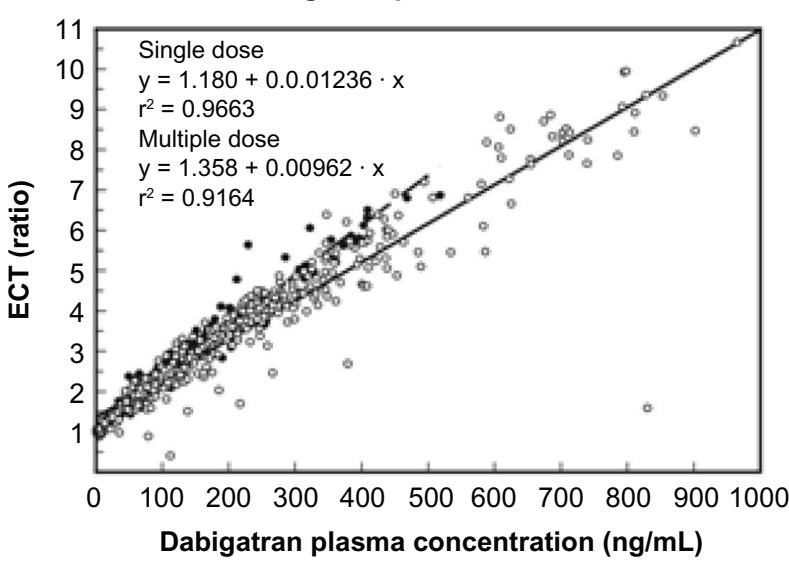

Figure 3 Responses of various coagulation assays to dabigatran in multiple studies.

Notes: Responses of various coagulation assays to dabigatran in plasma from healthy individuals from single dose $(\bullet)$ and multiple dose studies $(O)$, showing single dose (broken line) and multiple dose (solid line) correlation analysis results. Reprinted with permission Stangier J, Rathgen K, Stähle H, Gansser D, Roth W. The pharmacokinetics, pharmacodynamics and tolerability of dabigatran etexilate, a new oral direct thrombin inhibitor, in healthy male subjects. Br J Clin Pharmacol. 2007;64(3):292-303. Copyright @ 2007 John Wiley and Sons. ${ }^{15}$ Abbreviations: aPTT, activated partial thromboplastin time; INR, international normalized ratio; TT, thrombin time; ECT, Ecarin clotting time.

non inferior to warfarin $(P<0.001$ for non-inferiority; RR with dabigatran: 0.90 ; 95\% CI: $0.74-1.10)$, and at this dose there was a $20 \%$ reduction $(2.87 \%)$ in major bleeding risk compared with warfarin ( $P=0.003$ for superiority). A summary of these results can be seen in Figure 4.

Therefore dabigatran, at $150 \mathrm{mg}$ BID dose was superior to warfarin with respect to stroke or systemic embolism, and the $110 \mathrm{mg}$ BID dose was superior to warfarin with respect to major bleeding. The $150 \mathrm{mg}$ BID dosing had a near significant $(P=0.051)$ decrease in mortality (RR 0.88 , 95\% CI: 0.77-1.00). Based on these results, the ACCF/AHA/ HRS (American College of Cardiology Foundation/American Heart Association/Heart Rhythm Society) made a focused update in 2011 to the management of patients with AF; they gave dabigatran $150 \mathrm{mg}$ BID dosing a Class 1a indication as an alternative to warfarin for the prevention of stroke and systemic thromboembolism in patients with paroxysmal or permanent AF, and with risk factors for stroke and no absolute contraindication to anticoagulation. The 2012 focused updates on AF for both the Canadian Cardiovascular Society and the European Society of Cardiology made stronger statements, stating that NOACs, including dabigatran, were preferred to warfarin in most patients with $\mathrm{AF}^{26,27}$

\section{Key differences between dabigatran and warfarin}

The rates of intracranial hemorrhage in the RE-LY trial were $0.76 \%, 0.31 \%$, and $0.23 \%$ per year among those assigned to warfarin, dabigatran $150 \mathrm{mg}$, and dabigatran $110 \mathrm{mg}$, respectively ( $P<0.001$ for either dabigatran dose versus warfarin). This is equivalent to $59 \%$ and $70 \%$ reduction in intracranial bleeds for the $150 \mathrm{mg}$ and $110 \mathrm{mg}$ dose dabigatran, respectively. The case fatality of an intracranial bleed was $52 \%$, and despite a lack of a reversal agent, was not different between the groups. ${ }^{28}$ The mechanism for the lower rate of intracranial hemorrhage is unknown, but it appears to persist in the novel oral direct factor 


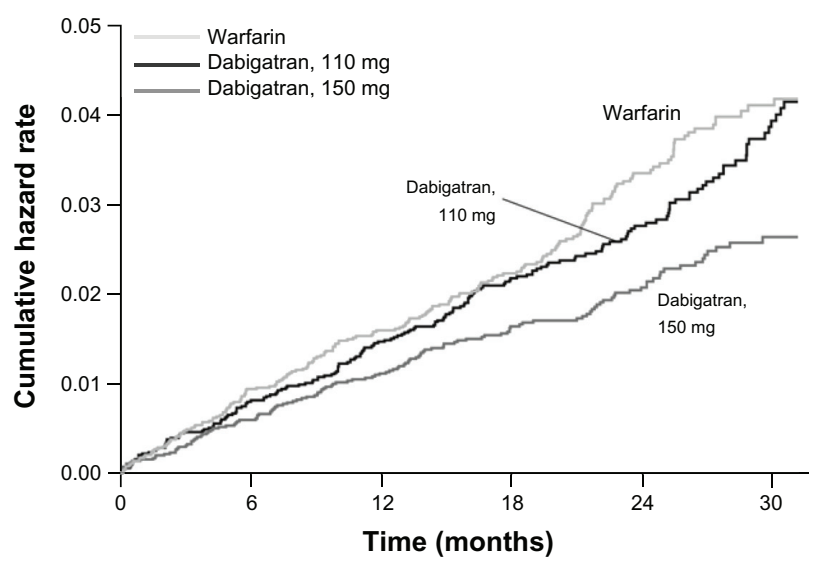

Figure 4 Efficacy of two doses of dabigatran in the RE-LY trial.

Notes: Efficacy of two doses of dabigatran (110 mg and $150 \mathrm{mg}$ bid) as cumulative hazard rates for stroke or systemic embolism (the primary outcome) in the Randomized Evaluation of Long Term Anticoagulant Therapy (RE-LY) trial by treatment. Reprinted with permission Connolly SJ, Ezekowitz MD, Yusuf S, et al. Dabigatran versus warfarin in patients with atrial fibrillation. $N$ Engl J Med. 2009;36 (12): I 139-1 I5I. Copyright (C 2009 Massachusetts Medical Society. ${ }^{25}$

Xa inhibitors (apixaban, rivaroxaban) as well. ${ }^{29,30}$ In patients younger than 75 years, both doses of dabigatran significantly reduced major bleeding complications compared with warfarin; however, in elderly patients ( $>75$ years), the lower dose was associated with a similar rate and the higher dose with an increased rate of major bleeding. ${ }^{31}$ The higher dose (150 mg BID) increased major gastrointestinal hemorrhage in all patients (RR 1.5, 95\% CI: $1.19-1.89, P<0.001)$, namely lower GI bleeding.

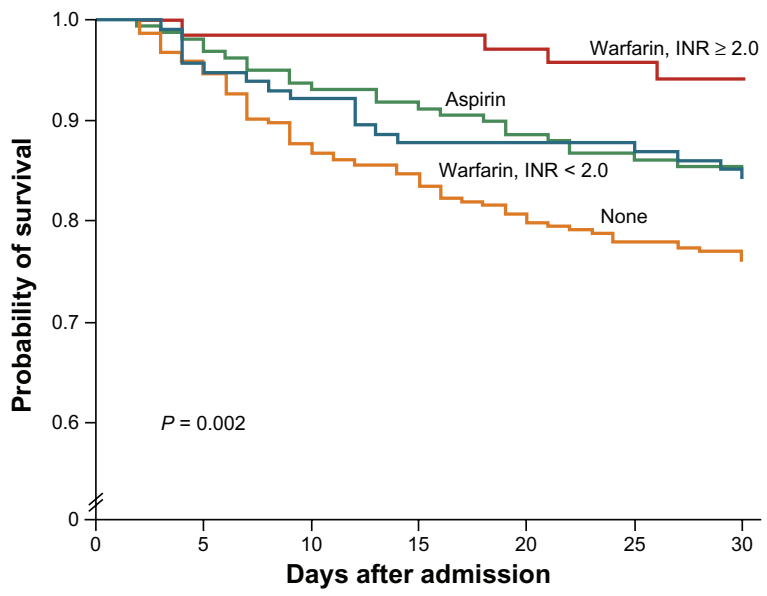

Figure 5 Kaplan-Meier estimates of survival in the 30 days after an ischemic stroke among patients with NVAF.

Notes: Figure shows Kaplan-Meier estimates of survival in the 30 days after an ischemic stroke among patients with NVAF, according to the antithromboticmedication status at admission. Patients who were taking warfarin are separated into two groups: those with an INR of less than 2.0, and those with an INR of 2.0 or greater. $P$-value $=0.002$ refers to the overall difference among these groups. From Hylek EM, Go AS, Chang Y. Effect of intensity of oral anticoagulation on stroke severity and mortality in atrial fibrillation. N Engl J Med. 349(II):1019-1026. Copyright () 2003 Massachusetts Medical Society. Reprinted with permission by Massachusetts Medical Society. ${ }^{58}$

Abbreviations: INR, international normalized ratio; NVAF, nonvalvular atrial fibrillation.
There was a numerical increase in myocardial infarction (MI) with both doses of dabigatran compared with warfarin; this result was reproduced in a metaanalysis of all trials including RE-LY. ${ }^{32}$ The addition of antiplatelet therapy in patients with $\mathrm{AF}$ and at high risk for acute coronary syndrome seems prudent, although there is no evidence that concomitant antiplatelet therapy provides any benefit in addition to dabigatran in reducing MI. ${ }^{33}$ Concomitant antiplatelet drugs are associated with increased rates of bleeding complications, without evidence for additional stroke protection. ${ }^{34}$ The ongoing trial RE-DEEM dose-finding study (NCT00621855) is evaluating the safety and efficacy of dabigatran etexilate in acute coronary syndromes. This study may provide insight into the optimal medical management in patients at high risk for ischemic cardiovascular events. Until we have more data, the benefits of dabigatran over warfarin for stroke prevention and the reduction in all-cause mortality appear to outweigh the small increase in the risk of MI. ${ }^{35}$

The most likely reason for discontinuation of dabigatran in RE-LY was GI distress or dyspepsia. This is presumably related to the tartaric acid content combined to assist in oral absorption. Dyspepsia occurred twice as frequently on both doses of dabigatran compared to warfarin (approximately $11.5 \%$ for both doses of dabigatran compared to $5.8 \%$ for warfarin). While proton pump inhibitors may ameliorate this side effect, they reduce the absorption of the drug. Dabigatran can probably be safely taken with meals, though there is limited experience. It is suggested that absorption of the drug may be delayed by 1 hour with improved tolerability as regards GI distress when taking the medication with meals.

An additional key difference between dose adjusted warfarin and oral dabigatran lies in the time in therapeutic range (TTR) for patients on warfarin. Guidelines recommend an INR target of 2.0-3.0 for patients on warfarin for stroke prevention in AF. Prior studies have shown that consistent INR levels below 2.0 are associated with an increased risk for embolic stroke, and levels higher than 3.5 with high rates of intra cranial hemorrhage. In the RE-LY trial, the advantages of dabigatran over warfarin were greater at sites with poor INR control than at those with good INR control. ${ }^{36}$ In patients felt to be at bleeding risk on oral anticoagulation, the use of 'low dose' warfarin should be discouraged.

\section{Differences between dosages}

Based on the results of this study, the United States Food and Drug Administration (FDA) approved the $150 \mathrm{mg}$ dosage but not the $110 \mathrm{mg}$ dosage. The decision was controversial and the $110 \mathrm{mg}$ BID dosing is still used outside the US. 
In a response published in the New England Journal, the FDA's decision was based upon the "inability to identify any subgroup in which use of the lower dose would not represent a substantial disadvantage". ${ }^{37}$ In patients with moderate renal impairment $(\mathrm{CrCl}>30-50 \mathrm{~mL} /$ minute $)$, the rate of stroke or systemic embolism with $150 \mathrm{mg}$ of dabigatran (1.3 per 100 patient-years) was approximately half that with $110 \mathrm{mg}$ (2.4 per 100 patient-years), and the rate of bleeding was no greater (5.3 versus 5.7 major bleeding episodes per 100 patient-years). In patients who were 75 years of age or older $(n=7238)$, the rate of stroke or systemic embolism was lower with $150 \mathrm{mg}$ of dabigatran (1.4 per 100 patient-years) than with $110 \mathrm{mg}$ (1.9 per 100 patient-years), but the rate of major bleeding was higher (5.1 versus 4.4 per 100 patient-years). In patients with a prior stroke or transient ischemic attack, the rates of stroke or embolism were increased; however, this result did not change the findings in outcomes. Namely, the $150 \mathrm{mg}$ dabigatran reduced stroke or systemic embolism and $110 \mathrm{mg}$ dabigatran was non inferior. ${ }^{38}$ Therefore, the FDA argued that because stroke and systemic embolism is a more clinically significant endpoint than major hemorrhage, any risk-benefit assessment would favor the higher dose. Indeed, in RE-LY, $57 \%$ of patients who had a major bleeding event during the study either resumed taking or had no interruption in their study medication, continuing to take the same dose. ${ }^{37}$ In RE-LY, patients randomized to dose-adjusted warfarin strategy were able to achieve therapeutic INR values (TTR) $64.4 \%$ of the time. At our institution (Vanderbilt University Medical Center/Vanderbilt Medical Group anticoagulation clinic, Nashville, TN, USA), using a pharmacogeconomic assisted approach, maximum TTR has been $77 \%$. This reflects an inherent weakness in a warfarin usage strategy.

Anticoagulation for AF is additionally known to be significantly underutilized, with only $60 \%-70 \%$ of qualified patients receiving anticoagulation. ${ }^{39,40}$ The low utilization of systemic anticoagulation results in higher stroke rates and overall medical costs (averaging $\$ 9836$ per patient per year). ${ }^{41}$ It has been suggested that some patients who currently reject warfarin for fear of bleeding risks might have been willing to use dabigatran at the $110 \mathrm{mg}$ dose, with its lower known bleeding risk. However, with the current data, it could be argued that such a strategy of use with a less-effective regimen would lead to unnecessary strokes and disability, and in the US, the $110 \mathrm{mg}$ dose is not available. The head to head superiority of dabigatran may be related to pharmacodynamic effects of its action directly on clot bound thrombin, or may simply reflect its superior pharmacokinetics, allowing for an essentially $100 \%$ 'therapeutic' dosage.

\section{Bleeding complications and reversal agents}

There is currently no specific antidote available to antagonize the anticoagulant effect of dabigatran. When bleeding occurs in the setting of dabigatran use, treatment options are primarily supportive. Given the relatively short half-life (12-14 hours), drug discontinuation suffices in most clinical situations. Dabigatran has a relatively low ( $35 \%)$ plasma protein binding, and dialysis can remove approximately $60 \%$ of the drug after 2-3 hours of dialysis. Activated charcoal may help remove unabsorbed drug from the GI tract if given within 2 hours of ingestion. In instances of life-threatening bleeding, nonspecific prohemostatic agents such as recombinant activated factor VII and prothrombin complex concentrates can be considered. A monoclonal antibody capable of rapidly and completely inhibiting dabigatran's anticoagulant activity is currently under development for clinical use.

Post market reports of bleeding and thrombotic events have raised concerns about the safety of dabigatran in the real world. Post marketing reports of bleeding in the AERS database are highly biased as bleeding complications are likely underreported for warfarin compared to events occurring with the more recently available dabigatran. Based on data from insurance claims and administrative data, the FDA issued a statement in November $2012^{56}$ that the rates of bleeding associated with dabigatran and warfarin were similar to those seen in the RE-LY trial for patients starting either drug for the first time. For the populations in the Mini-Sentinel data assessment, bleeding complications were roughly twice as common in new use of warfarin compared to dabigatran. Individual practitioner fears over the lack of an available reversal agent for dabigatran must be carefully balanced with the evidence from RE-LY showing an overall reduction in major bleeding risk, with no increased death, for patients on BID dabigatran.

\section{Use of dabigatran in patients undergoing urgent surgical or invasive procedures}

Perioperative dabigatran usage in the invasive procedure setting may potentially be an improvement over typical low molecular weight heparin (LMWH) bridging for patients on traditional warfarin. Warfarin patients are often exposed to 1-2 weeks of variable interruptions in systemic anticoagulation perioperatively. Healey et al recently undertook an analysis of patients in the RE-LY trial undergoing invasive procedures. ${ }^{42}$ The lack of an available reversal agent for 
dabigatran has led to fears of increased major bleeding events with invasive procedures. The investigators evaluated bleeding rates from 7 days before, until 30 days after invasive procedures. A total of 4591 patients underwent one or more invasive procedures in RE-LY; $24.7 \%$ of patients were on dabigatran $110 \mathrm{mg}, 25.4 \%$ on dabigatran $150 \mathrm{mg}$, and $25.9 \%$ received warfarin, $P=0.34$.

Procedures included pacemaker/defibrillator insertion (10.3\%), dental procedures $(10.0 \%)$, diagnostic procedures $(10.0 \%)$, cataract removal $(9.3 \%)$, colonoscopy $(8.6 \%)$, and joint replacement (6.2\%). Among patients assigned to either dabigatran dose, the last dose of study drug was given a mean of 49 (35-85) hours before the procedure. In comparison, patients receiving oral warfarin were off anticoagulation an average of 114 hours (87-144 hours), $P<0.001$. There were no differences in the rates of periprocedural major bleeding between patients receiving dabigatran $110 \mathrm{mg}$ (3.8\%), dabigatran $150 \mathrm{mg}(5.1 \%)$, or warfarin (4.6\%). Among patients having urgent surgery there was a trend in favor of dabigatran (major bleeding occurred in 17.8\% with dabigatran $110 \mathrm{mg}$, $17.7 \%$ with dabigatran $150 \mathrm{mg}$, and $21.6 \%$ with warfarin: dabigatran $110 \mathrm{mg}$; relative risk, 0.82 ; 95\% CI, 0.48-1.41; $P=0.47$; dabigatran $150 \mathrm{mg}$ : relative risk, $0.82 ; 95 \%$ CI, 0.50-1.35; $P=0.44)$.

In this study, dabigatran and warfarin were associated with similar rates of periprocedural bleeding, including patients having urgent surgery. When factoring in the costs associated with LMWH bridging, and the potential transient increase in stroke risk associated with a longer time withholding anticoagulation with warfarin, dabigatran use appears favorable for patients requiring invasive procedures. ${ }^{42}$

Results of a formal randomized trial of bridging warfarin versus continuous oral anticoagulation (BRUISE-CONTROL) specifically in cardiac device implant procedures done at the University of Ottawa (Ottawa, Canada) should be available soon (Principal Applicant: David Birnie, MD). The trial was stopped early at a specified second interim analysis in March 2013 (ClinicalTrials.gov identifier NCT00800137).

\section{Use of dabigatran in the setting of AF or left atrial catheter ablation procedures}

Radiofrequency catheter ablation for AF has evolved to a primary treatment modality for many patients with drug refractory atrial arrhythmias. Intra procedural thrombosis on vascular access wires, mapping and ablation catheters, and transseptal sheaths, can lead to acute periprocedural cerebral embolization. Atrial inflammation and endothelial left atrial damage from thermal injury may lead to delayed thrombus formation in the left atrium or left atrial appendage, and necessitates maintaining therapeutic anticoagulation for a minimum of 2 months post ablation, according to ACCF/ AHA/HRS 2012 updates on AF. These guidelines do not elaborate on the use of dabigatran during catheter ablation, citing little available evidence. Recent studies have demonstrated that performing AF ablation while fully anticoagulated on dose adjusted warfarin (intraoperative INR 2.0-3.0) is safe; it is also associated with lower bleeding complications than a heparin or low molecular weight heparin bridging strategy. ${ }^{43,44}$ Data on the use of dabigatran for periprocedural anticoagulation are limited, but several recent studies show this may be safely used and that it can simplify issues with maintaining therapeutic systemic anticoagulation periprocedurally.

A study by Winkle et al reported the "safe use of dabigatran after AF ablation". ${ }^{44}$ Their study involved 123 consecutive patients undergoing AF ablation, approximately one-fourth of the patients (34 patients) were taking dabigatran before the procedure. Dabigatran was stopped for two doses (24 hours) prior to the procedure in patients with normal renal function. During the ablation, however, the target ACT was 225 seconds, which is lower than typical, with most accepted protocols targeting a higher ACT (300-400 seconds). Protamine was used as a reversal agent and enoxaparin $(0.5 \mathrm{mg} / \mathrm{kg})$ was given immediately post procedure, with dabigatran restarted 10 hours after a second enoxaparin injection. Winkle et al reported no bleeding or thromboembolic complications. ${ }^{44}$

A 2012 multicenter observational study by Lakkireddy et al involving 145 patients taking dabigatran reported a significantly higher risk of major bleeding ( $6 \%$ versus $1 \%)$, total bleeding (14\% versus $6 \%$ ), and composite risk of bleeding or thromboembolic event (16\% versus $6 \%$ ) with the use of periprocedural anticoagulation with dabigatran compared to therapeutic dose-adjusted warfarin. ${ }^{45}$ It should be emphasized that dabigatran was not stopped until the morning of the procedure (in contrast to guidelines in the USPI for dabigatran), and was subsequently restarted 3 hours after transseptal sheaths were pulled. Given that the half-life of dabigatran is 12-17 hours in patients with normal renal function, this protocol was nearly equivalent to performing the procedure on uninterrupted dabigatran.

We performed a retrospective cohort study on 254 patients who underwent left atrial catheter ablation for AF or left atrial flutter at Vanderbilt Heart and Vascular Institute from 2011 through 2012. ${ }^{46}$ Periprocedural anticoagulation utilized dabigatran in 122 patients and warfarin in 135 patients. Three late thromboembolic complications occurred in the dabigatran 
group $(2.5 \%)$, compared with one $(0.7 \%)$ in the warfarin group $(P=0.28)$. The dabigatran group had a similar minor bleeding $(2.5 \%$ versus $7.4 \%, P=0.07)$, major bleeding $(1.6 \%$ versus $0.7 \%, P=0.51$ ), and composite of bleeding and thromboembolic complications $(6.6 \%$ versus $8.9 \%, P=0.49)$ when compared to warfarin. There were no acute thromboembolic complications in either group ( $<24$ hours post ablation). In our study, dabigatran was held 24-30 hours pre-procedure, and restarted 4-6 hours after hemostasis was achieved.

Similar results were recently published by the University of Michigan group using case-control analysis; the study looked at 763 consecutive patients (mean age 61 years \pm 10 years) undergoing AF ablation using dabigatran $(\mathrm{n}=191)$ or uninterrupted warfarin $(n=572)$ for periprocedural anticoagulation. ${ }^{47}$ There were no thromboembolic complications in either group. The prevalence of major ( 4 of 191, 2.1\%) and minor (5 of 191, $2.6 \%$ ) bleeding complications in the dabigatran group was similar to that in the warfarin group (12 of 572, 2.1\%; $P=1.0$ and 19 of $572,3.3 \% ; P=0.8$, respectively).

Though currently no prospective randomized data are available, it seems that the majority of bleeding risk acutely from left atrial catheter ablation is likely attributable to the mechanical complications of the procedure, rather than the choice of periprocedural anticoagulant. The prevention of acute periprocedural stroke or embolism is dependent on tight ACT monitoring of typically an intravenous heparin infusion (goal ACT 300-400 seconds). The effect of dabigatran on the ACT is not well established and could cause under dosing of heparin intra operatively. This is the main rationale for holding dabigatran 24 hours prior to left atrial ablation at our institution. The prevention of late post ablation embolic events depends on maintaining tight continuous systemic anticoagulation, for which dabigatran's pharmacokinetics are ideal.

\section{Use of dabigatran in the setting of elective percutaneous coronary intervention}

Limited clinical data on the utility of dabigatran in the setting of elective percutaneous coronary intervention (PCI) are available. One small recent study suggests that dabigatran may be a poor choice, with a high risk of requiring bailout anticoagulation for acute procedure-related MI during elective coronary stent placement. The study by Vranckx et al is a randomized study of dabigatran in elective PCI, in patients with stable coronary artery disease. ${ }^{48}$ Patients on long-term anticoagulant treatment with dabigatran undergoing a PCI were studied. Markers of coagulation activation during elective PCI were obtained intra-procedure to determine if PCI coagulation activation was adequately suppressed by dabigatran without additional heparin treatment. Fifty patients on dual antiplatelet therapy were randomized $(2: 2: 1)$ to either pre-procedural dabigatran $110 \mathrm{mg}$ BID $(\mathrm{n}=19)$ or $150 \mathrm{mg}$ BID ( $\mathrm{n}=21)$, as compared to standard intra procedural unfractionated heparin (UFH) $(n=10)$.

Post PCI, levels of prothrombin fragment $1+2$ $(\mathrm{F} 1+2)$, with increased thrombin-antithrombin complexes were observed in the dabigatran patients, with no elevation in the heparin group. Five out of 40 (12.5\%) patients required bailout anticoagulation in the dabigatran group, and four experienced a procedural MI, versus one out of ten in the UFH group, who had a stent thrombosis without MI prior to the study PCI. As previously mentioned, the RE-DEEM trial is ongoing and may shed further light on this issue in the setting of acute coronary syndromes, when urgent PCI will be performed during the highly thrombogenic period following coronary plaque rupture. ${ }^{35,48}$

\section{Switching between dabigatran and warfarin}

As per protocol in the RE-LY and PETRO trials of dabigatran, the package insert for patients previously on stable warfarin dosing and switching to dabigatran recommends starting dabigatran when the INR drifts below 2.0. ${ }^{25,49}$ When switching from dabigatran to warfarin in patients with normal renal function ( $\mathrm{CrCl}<50 \mathrm{~mL} /$ minute), warfarin should be started 3 days before discontinuing dabigatran. In patients with renal dysfunction, warfarin should be started 2 days before and 1 day before discontinuing dabigatran in patients with moderate dysfunction $(\mathrm{CrCl} 30-50 \mathrm{~mL} /$ minute $)$ and severe ( $\mathrm{CrCl} 15-30 \mathrm{~mL} / \mathrm{minute})$ renal dysfunction, respectively.

\section{Use of dabigatran in patients undergoing cardioversion}

Cardioversion (both electric and pharmacological) in patients with AF is associated with an increased risk of thromboembolic events. ${ }^{50,51}$ The risk of thromboembolism is highest during the first week after cardioversion, with rates of approximately $5 \%$ in the absence of adequate anticoagulation. ${ }^{52}$ Current guidelines recommend that patients with $\mathrm{AF}$ of greater than 48 hours duration have therapeutic anticoagulation for at least 3 weeks before and 4 weeks after cardioversion. ${ }^{53}$

In the RE-LY trial, a total of 1983 cardioversions resulted in thromboembolic rates of $0.8 \%, 0.3 \%$, and $0.6 \%$ for the dabigatran $110 \mathrm{mg}$, dabigatran $150 \mathrm{mg}$, and warfarin treatment arms, respectively. ${ }^{54}$ Therefore dabigatran appears to be a reasonable alternative to warfarin in patients requiring 
cardioversion. Since warfarin's delayed onset of action may require the use of heparin bridging, dabigatran has the advantage of being more suitable for outpatient management, and its use may prove economical by avoiding hospitalization.

\section{Use of dabigatran in patients with mechanical heart valves}

An exclusion to entry in the RE-LY study was the presence of a prosthetic heart valve, or hemodynamically significant heart valve disease (heart valve disease sufficient that it would be expected surgical replacement is warranted within 1-2 years,) thus the repetitive labeling and marketing of dabigatran for use in patients with "non-valvular AF". A randomized study was designed and the trial methods published by Van de Werf et al in $2012 .{ }^{55}$ The trial was designed as a 2:1 dabigatran to warfarin therapy for patients undergoing implantation of a mechanical bileaflet valve (aortic or mitral or both) or having undergone implantation of a mitral bileaflet valve $>3$ months before randomization. Dabigatran was given as either a $150 \mathrm{mg}$ BID or a $75 \mathrm{mg}$ BID dosing based on renal clearance. Warfarin management and target INR was according to current practice guidelines and the discretion of the treating physicians. Note the $75 \mathrm{mg}$ BID dosing is based on pharmacokinetics of the drug, and was not formally studied in the RE-LY trial.

Referred to as the RE-ALIGN trial, enrollment began in December 2011. The study was halted by the manufacturer in December 2012. In September 2012, as reported by "heartwire", Canadian physicians reported cases of two women who had undergone valve replacement years prior and were stable on warfarin, but subsequently suffered valve thrombosis when switched to dabigatran. Dosing in the REALIGN trial included $150 \mathrm{mg}, 220 \mathrm{mg}$, and $300 \mathrm{mg}$ BID dosing with a control group on dose adjusted warfarin therapy. There were notably increased bleeding risks following heart valve surgery in the dabigatran groups compared to the warfarin group, in addition to the increased risk for heart valve thrombosis. The FDA and Boehringer-Ingelheim (Ingelheim, Germany) have since made efforts to reinforce the warning labels for dabigatran to alert physicians that dabigatran should not be used for anticoagulation in patients with prosthetic heart valves, particularly mechanical bileaflet prostheses.

\section{Post market performance of dabigatran as regards bleeding risks}

Post marketing reports of bleeding with dabigatran led to questions in the mainstream media about the risk-benefit profile of dabigatran as described in its labeling (based on the RE-LY trial data). The RE-LY trial does demonstrate that bleeding (particularly lower GI bleeding and intracranial bleeding) was a potential serious side effect of dabigatran use. Despite this, high numbers of early reports of adverse bleeding led the FDA to review reports received by FAERS (FDA adverse event reporting system). These reports were recently reviewed by Southworth et al, who found the reports of bleeding generally did not include information on patients' risk factors, age, renal function, or cause of death. There was no indication that the drug was being commonly used outside its FDA approved indications. ${ }^{56}$

Southworth et al went on to compare bleeding rates for dabigatran and warfarin using insurance claim data and administrative data from the FDA Mini-Sentinel database. A database query from October 19, 2010 (the date of dabigatran approval) to December 31, 2011, was performed to identify inpatient diagnosis codes for intracranial and gastrointestinal hemorrhages associated with new use of dabigatran or warfarin. The authors found that bleeding rates associated with dabigatran use during the period of interest did not appear to be higher than those associated with warfarin. They go on to conclude that the apparent increased adverse bleeding reports with dabigatran were likely the result of "stimulated" reporting, due to public attention drawn to bleeding risks with a novel agent. Formal post marketing surveillance studies are ongoing. ${ }^{56}$

\section{Conclusion}

Dabigatran etexilate was the first FDA approved version of a novel class of oral anticoagulants known as NOACs. It has become a widely available treatment alternative to dose adjusted warfarin for stroke prevention in AF. Large randomized clinical trial data show its superiority to warfarin in selected patients with NVAF to prevent thromboembolic complications, with an improved safety profile compared to warfarin. The drug has rapid onset to full anticoagulation (within 2 hours), has a predictable therapeutic window allowing BID dosing without routine monitoring, there are minimal drug-drug interactions, and no diet restrictions for patients taking dabigatran. Dabigatran does have potential increased risks of gastrointestinal hemorrhage, higher myocardial infarction rate, concerns about dyspepsia, and lacks the ability to rapidly reverse its anticoagulant effect in the setting of life-threatening adverse bleeding. It is a safe and effective option for periprocedural anticoagulation in patients undergoing left atrial catheter ablation. It should not be used in patients with mechanical prosthetic heart valves or end stage renal disease. Patients who cannot adhere to BID dosing, are prone to intestinal bleeding, or are at risk for wide fluctuations in renal function, are poor candidates for the drug. 


\section{Disclosure}

The authors report no conflicts of interest in this work.

\section{References}

1. Coppens M, Eikelboom JW, Gustafsson D, Weitz JI, Hirsh J. Translational success stories: development of direct thrombin inhibitors. Circ Res. 2012;111(7):920-929.

2. Olsson SB; Executive Steering Committee of the SPORTIF III Investigators. Stroke prevention with the oral direct thrombin inhibitor ximelagatran compared with warfarin in patients with non-valvular atrial fibrillation (SPORTIF III): randomised controlled trial. Lancet. 2003;362(9397):1691-1698.

3. Mohapatra R, Tran M, Gore JM, Spencer FA. A review of the oral direct thrombin inhibitor ximelagatran: not yet the end of the warfarin era. Am Heart J. 2005;150(1):19-26.

4. Bates SM, Weitz JI. The mechanism of action of thrombin inhibitors. J Invasive Cardiol. 2000;12 Suppl F:27F-32F.

5. Eriksson BI, Quinlan DJ, Eikelboom JW. Novel oral factor Xa and thrombin inhibitors in the management of thromboembolism. Annu Rev Med. 2011;62:41-57.

6. Becker RC. Cell-based models of coagulation: a paradigm in evolution. J Thromb Thrombolysis. 2005;20(1):65-68.

7. Weitz JI, Hudoba M, Massel D, Maraganore J, Hirsh J. Clot-bound thrombin is protected from inhibition by heparin-antithrombin III but is susceptible to inactivation by antithrombin III-independent inhibitors. J Clin Invest. 1990;86(2):385-391.

8. Weitz JI, Leslie B, Hudoba M. Thrombin binds to soluble fibrin degradation products where it is protected from inhibition by heparin-antithrombin but susceptible to inactivation by antithrombin-independent inhibitors. Circulation. 1998;97(6):544-552.

9. Vanassche T, Verhaegen J, Peetermans WE, et al. Inhibition of staphylothrombin by dabigatran reduces Staphylococcus aureus virulence. J Thromb Haemost. 2011;9(12):2436-2446.

10. Xiao Z, Théroux P. Platelet activation with unfractionated heparin at therapeutic concentrations and comparisons with a low-molecularweight heparin and with a direct thrombin inhibitor. Circulation. 1998;97(3):251-256.

11. Ansell J. Warfarin versus new agents: interpreting the data. Hematology Am Soc Hematol Educ Program. 2010;2010:221-228.

12. Wrigley BJ, Lip GY, Shantsila E. Novel oral anticoagulants: the potential relegation of vitamin $\mathrm{K}$ antagonists in clinical practice. Int J Clin Pract. 2010;64(7):835-838.

13. Stangier J. Clinical pharmacokinetics and pharmacodynamics of the oral direct thrombin inhibitor dabigatran etexilate. Clin Pharmacokinet. 2008;47(5):285-295.

14. Stangier J, Eriksson BI, Dahl OE, et al. Pharmacokinetic profile of the oral direct thrombin inhibitor dabigatran etexilate in healthy volunteers and patients undergoing total hip replacement. J Clin Pharmacol. 2005;45(5):555-563.

15. Stangier J, Rathgen K, Stähle H, Gansser D, Roth W. The pharmacokinetics, pharmacodynamics and tolerability of dabigatran etexilate, a new oral direct thrombin inhibitor, in healthy male subjects. Br J Clin Pharmacol. 2007;64(3):292-303.

16. Eisert WG, Hauel N, Stangier J, Wienen W, Clemens A, van Ryn J. Dabigatran: an oral novel potent reversible nonpeptide inhibitor of thrombin. Arterioscler Thromb Vasc Biol. 2010;30(10):1885-1889.

17. Stangier J, Rathgen K, Stähle H, Mazur D. Influence of renal impairment on the pharmacokinetics and pharmacodynamics of oral dabigatran etexilate: an open-label, parallel-group, single-centre study. Clin Pharmacokinet. 2010;49(4):259-268.

18. Wann LS, Curtis AB, Ellenbogen KA, et al. 2011 ACCF/AHA/HRS focused update on the management of patients with atrial fibrillation (update on dabigatran): a report of the American College of Cardiology Foundation/American Heart Association Task Force on practice guidelines. J Am Coll Cardiol. 2011;57(11):1330-1337.
19. Blech S, Ebner T, Ludwig-Schwellinger E, Stangier J, Roth W. The metabolism and disposition of the oral direct thrombin inhibitor, dabigatran, in humans. Drug Metab Dispos. 2008;36(2):386-399.

20. Stangier J, Stähle H, Rathgen K, Roth W, Shakeri-Nejad K. Pharmacokinetics and pharmacodynamics of dabigatran etexilate, an oral direct thrombin inhibitor, are not affected by moderate hepatic impairment. J Clin Pharmacol. 2008;48(12):1411-1419.

21. Nutescu E, Chuatrisorn I, Hellenbart E. Drug and dietary interactions of warfarin and novel oral anticoagulants: an update. J Thromb Thrombolysis. 2011;31(3):326-343.

22. Stangier J, Stähle H, Rathgen K, Fuhr R. Pharmacokinetics and pharmacodynamics of the direct oral thrombin inhibitor dabigatran in healthy elderly subjects. Clin Pharmacokinet. 2008;47(1):47-59.

23. van Ryn J, Stangier J, Haertter S, et al. Dabigatran etexilate - a novel, reversible, oral direct thrombin inhibitor: interpretation of coagulation assays and reversal of anticoagulant activity. Thromb Haemost. 2010;103(6):1116-1127.

24. Stangier J, Feuring M. Using the HEMOCLOT direct thrombin inhibitor assay to determine plasma concentrations of dabigatran. Blood Coagul Fibrinolysis. 2012;23(2):138-143.

25. Connolly SJ, Ezekowitz MD, Yusuf S, et al. Dabigatran versus warfarin in patients with atrial fibrillation. $N$ Engl J Med. 2009;361(12): $1139-1151$.

26. Skanes AC, Healey JS, Cairns JA, et al. Focused 2012 update of the Canadian Cardiovascular Society atrial fibrillation guidelines: recommendations for stroke prevention and rate/rhythm control. Can J Cardiol. 2012;28(2):125-136.

27. Camm AJ, Lip GY, De Caterina R, et al; ESC Committee for Practice Guidelines-(PG); Document Reviewers 2012 focused update of the ESC Guidelines for the management of atrial fibrillation: an update of the 2010 ESC Guidelines for the management of atrial fibrillation. Developed with the special contribution of the European Heart Rhythm Association. Eur Heart J. 2012;33(21):2719-2747.

28. Hart RG, Diener HC, Yang S, et al. Intracranial hemorrhage in atrial fibrillation patients during anticoagulation with warfarin or dabigatran: the RE-LY trial. Stroke. 2012;43(6):1511-1517.

29. Granger CB, Alexander JH, McMurray JJ, et al. Apixaban versus warfarin in patients with atrial fibrillation. $N$ Engl J Med. 2011;365(11):981-992.

30. Patel MR, Mahaffey KW, Garg J, et al; ROCKET AF Investigators. Rivaroxaban versus warfarin in nonvalvular atrial fibrillation. $N$ Engl J Med. 2011;365(10):883-891.

31. Eikelboom JW, Wallentin L, Connolly SJ, et al. Risk of bleeding with 2 doses of dabigatran compared with warfarin in older and younger patients with atrial fibrillation: an analysis of the randomized evaluation of long-term anticoagulant therapy (RE-LY) trial. Circulation. 2011;123(21):2363-2372.

32. Uchino K, Hernandez AV. Dabigatran association with higher risk of acute coronary events: meta-analysis of noninferiority randomized controlled trials. Arch Intern Med. 2012;172(5):397-402.

33. Hohnloser SH, Oldgren J, Yang S, et al. Myocardial ischemic events in patients with atrial fibrillation treated with dabigatran or warfarin in the RE-LY (Randomized Evaluation of Long-Term Anticoagulation Therapy) trial. Circulation. 2012;125(5):669-676.

34. Dans AL, Connolly SJ, Wallentin L, et al. Concomitant use of antiplatelet therapy with dabigatran or warfarin in the randomized evaluation of long-term anticoagulation therapy (RE-LY) trial. Circulation. 2013;127(5):634-640.

35. Eikelboom JW, Weitz JI. Anticoagulation therapy. Dabigatran and risk of myocardial infarction. Nat Rev Cardiol. 2012;9(5):260-262.

36. Wallentin L, Yusuf S, Ezekowitz MD, et al; RE-LY investigators. Efficacy and safety of dabigatran compared with warfarin at different levels of international normalised ratio control for stroke prevention in atrial fibrillation: an analysis of the RE-LY trial. Lancet. 2010;376(9745):975-983.

37. Beasley BN, Unger EF, Temple R. Anticoagulant options - why the FDA approved a higher but not a lower dose of dabigatran. $N$ Engl $J$ Med. 2011;364(19):1788-1790. 
38. Diener HC, Connolly SJ, Ezekowitz MD, et al; RE-LY study group. Dabigatran compared with warfarin in patients with atrial fibrillation and previous transient ischaemic attack or stroke: a subgroup analysis of the RE-LY trial. Lancet Neurol. 2010;9(12):1157-1163.

39. Birman-Deych E, Radford MJ, Nilasena DS, Gage BF. Use and effectiveness of warfarin in Medicare beneficiaries with atrial fibrillation. Stroke. 2006;37(4):1070-1074.

40. Raji MA, Lowery M, Lin YL, Kuo YF, Baillargeon J, Goodwin JS. National utilization patterns of warfarin use in older patients with atrial fibrillation: a population-based study of Medicare Part D beneficiaries. Ann Pharmacother. 2013;47(1):35-42.

41. Mercaldi CJ, Ciarametaro M, Hahn B, et al. Cost efficiency of anticoagulation with warfarin to prevent stroke in medicare beneficiaries with nonvalvular atrial fibrillation. Stroke. 2011;42(1):112-118.

42. Healey JS, Eikelboom J, Douketis J, et al; RE-LY Investigators. Periprocedural bleeding and thromboembolic events with dabigatran compared with warfarin: results from the Randomized Evaluation of Long-Term Anticoagulation Therapy (RE-LY) randomized trial. Circulation. 2012;126(3):343-348.

43. Wazni OM, Beheiry S, Fahmy T, et al. Atrial fibrillation ablation in patients with therapeutic international normalized ratio: comparison of strategies of anticoagulation management in the periprocedural period. Circulation. 2007;116(22):2531-2534.

44. Winkle RA, Mead RH, Engel G, Kong MH, Patrawala RA. The use of dabigatran immediately after atrial fibrillation ablation. J Cardiovasc Electrophysiol. 2012;23(3):264-268.

45. Lakkireddy D, Reddy YM, Di Biase L, et al. Feasibility and safety of dabigatran versus warfarin for periprocedural anticoagulation in patients undergoing radiofrequency ablation for atrial fibrillation: results from a multicenter prospective registry. $J$ Am Coll Cardiol. 2012;59(13): $1168-1174$.

46. Kaiser DW, Streur MM, Nagarakanti R, Whalen SP, Ellis CR. Continuous warfarin versus peri-procedural dabigatran to reduce stroke and systemic embolism in patients undergoing catheter ablation for atrial fibrillation or left atrial flutter. J Interv Card Electrophysiol. [Epub April 28, 2013.]

47. Kim JS, She F, Jongnarangsin K, et al. Dabigatran vs warfarin for radiofrequency catheter ablation of atrial fibrillation. Heart Rhythm. 2013;10(4):483-489.

48. Vranckx P, Verheugt FW, de Maat MP, et al. A randomised study of dabigatran in elective percutaneous coronary intervention in stable coronary artery disease patients. Euro Intervention. 2013;8(9):1052-1060.
49. Ezekowitz MD, Reilly PA, Nehmiz G, et al. Dabigatran with or without concomitant aspirin compared with warfarin alone in patients with nonvalvular atrial fibrillation (PETRO Study). Am J Cardiol. 2007;100(9): 1419-1426.

50. Lown B, Perlroth MG, Kaidbey S, Abe T, Harken DE. "Cardioversion" of atrial fibrillation. A report on the treatment of 65 episodes in 50 patients. N Engl J Med. 1963;269:325-331.

51. Jensen JB, Humphries JO, Kouwenhoven WB, Jude JR. Electroshock for atrial flutter and atrial fibrillation. Follow-up studies on 50 patients. JAMA. 1965;194(11):1181-1184.

52. Bjerkelund CJ, Orning OM. The efficacy of anticoagulant therapy in preventing embolism related to DC electrical conversion of atrial fibrillation. Am J Cardiol. 1969;23(2):208-216.

53. Fuster V, Rydén LE, Cannom DS, et al; American College of Cardiology/American Heart Association Task Force on Practice Guidelines; European Society of Cardiology Committee for Practice Guidelines; European Heart Rhythm Association; Heart Rhythm Society. ACC/ AHA/ESC 2006 Guidelines for the Management of Patients with Atrial Fibrillation: a report of the American College of Cardiology/American Heart Association Task Force on Practice Guidelines and the European Society of Cardiology Committee for Practice Guidelines (Writing Committee to Revise the 2001 Guidelines for the Management of Patients With Atrial Fibrillation): developed in collaboration with the European Heart Rhythm Association and the Heart Rhythm Society. Circulation. 2006;114(7):e257-e354.

54. Nagarakanti R, Ezekowitz MD, Oldgren J, et al. Dabigatran versus warfarin in patients with atrial fibrillation: an analysis of patients undergoing cardioversion. Circulation. 2011;123(2):131-136.

55. Van de Werf F, Brueckmann M, Connolly SJ, et al. A comparison of dabigatran etexilate with warfarin in patients with mechanical heart valves: THE Randomized, phase II study to evaluate the safety and pharmacokinetics of oral dabigatran etexilate in patients after heart valve replacement (RE-ALIGN). Am Heart J. 2012;163(6):931-937.

56. Southworth MR, Reichman ME, Unger EF. Dabigatran and postmarketing reports of bleeding. $N$ Engl J Med. 2013;368(14):1272-1274.

57. Nagarakanti R, Ellis CR. Dabigatran in clinical practice. Clin Ther. 2012;34(10):2051-2060.

58. Hylek EM, Go AS, Chang Y. Effect of intensity of oral anticoagulation on stroke severity and mortality in atrial fibrillation. $N$ Engl J Med. 2003;349(11):1019-1026.
Vascular Health and Risk Management

\section{Publish your work in this journal}

Vascular Health and Risk Management is an international, peerreviewed journal of therapeutics and risk management, focusing on concise rapid reporting of clinical studies on the processes involved in the maintenance of vascular health; the monitoring, prevention and treatment of vascular disease and its sequelae; and the involvement of

\section{Dovepress}

metabolic disorders, particularly diabetes. This journal is indexed on PubMed Central and MedLine. The manuscript management system is completely online and includes a very quick and fair peer-review system, which is all easy to use. Visit http://www.dovepress.com/ testimonials.php to read real quotes from published authors. 